\title{
Optimal usage of cone beam computed tomography system with different field of views in image guided radiotherapy (IGRT)
}

\author{
Narayana Venkata Naga Madhusudhana Sresty', Thogata Ramanjappa², Alluri Krishnam Raju1, \\ Adavala Mallikarjuna ${ }^{1}$, Anil kumar Talluri ${ }^{1}$, Bhudevi Soubhagya ${ }^{1}$ \\ ${ }^{I}$ Department of Radiotherapy, Basavatarakam Indo American Cancer Institute and Research Centre, Hyderabad, India \\ ${ }^{2}$ Department of Physics, Sri Krishnadevaraya University, Anantapur, Andra Pradesh, India
}

Received January 27, 2015; Revised April 07, 2015; Accepted May 12, 2015; Published Online May 25, 2015

\section{Technical Report}

\begin{abstract}
Purpose: To find methods for optimal usage of XVI (X-ray volume imaging) system in Elekta synergy linear accelerator with different field of views for same lesion in order to minimize patient dose due to imaging. Methods: 20 scans of 2 individual patients with ca sigmoid colon and ca lung were used in this study. Kilo voltage collimators with medium field of view were used as per the preset information. Images were reconstructed for another collimator with small field of view. The set up errors were evaluated with XVI software. Shift results of both methods were compared. Results: Variation in treatment set up errors with M20 and S20 collimators were $\leq 0.2 \mathrm{~mm}$ in translational and $0.3^{0}$ in rotational shifts. Results showed almost equal translational and rotational shifts in both medium and small field of views with different collimators in all the scans. Visualization of target and surrounding structures were good enough and sufficient for XVI auto matching. Conclusion: Imaging with small field of view results less patient dose compared with medium or large field of views. It is Suggestible to use collimators with small field of view wherever possible. In this study, collimators with small field of view were sufficient for both patients though the preset information indicated medium field of view. But, it always depends on the area required for matching purpose. So, individual selection is important than preset information in the XVI system.
\end{abstract}

Keywords: XVI; Field of View; kV Collimator

\section{Introduction}

One of the main requirements for the tumor control in radiotherapy is, reproducible positioning of the patient in the treatment room as per the prior plan. This can be checked and corrected with the imaging systems integrated with the treatment delivery system. ${ }^{1}$ Cone beam computerised tomography (CBCT) integrated with linear accelerator for patient position verification is one of the prime methods in image guidance. ${ }^{2}$

It is now possible to compare full three-dimensional (3D) volumetric information with a high resolution and quality using CBCT in image guided radiotherapy (IGRT). Linear accelerator consists of an extendable X-ray tube fitted to the drum of it at orthogonal to MV treatment source and a motorized amorphous-Silicon flat imager panel opposite to it, is capable for this type of volumetric comparison. This $\mathrm{kV}$ imaging system is called X-ray Volumetric Imaging system (XVI). The XVI is sharing a common axis of rotation with the MV treatment source. Images can be acquired with gantry rotation with continuous X-ray exposure. During the rotation, the system will acquire planar projections which are used to make a three dimensional volume image. The rotation takes approximately two minutes. To handle raw cone-beam data, the most widely used algorithm for image reconstruction is a Feldkamp-type algorithm ${ }^{3,4}$ which is also the case for the XVI system.

Reconstructed volume images are registered to CT reference data set either manually or automatically. This gives six values for positional translation and rotation corrections which can be converted to table movements. This form of XVI system made IGRT, a better three-dimensional (3D) technique to improve daily patient treatment delivery. ${ }^{5,6,7,8}$ XVI system specifies different $\mathrm{kV}$ collimators for different sites of the patient. Though, there is a benefit, we should use this system optimally by scanning as less volume of the patient as possible. Since dose is added over a field wider than target zone, this has already influenced the debate on incidence of secondary cancers. ${ }^{9}$ Daily CBCT potentially increase secondary cancer risk up to $2-4 \% .{ }^{10}$ CBCT volume scan can be obtained with different field of views in XVI system namely small, medium and large. Scan volume will be less in small field of view. Our objective in this study is to compare the shift re- 
sults with different field of view selections for the optimal usage of CBCT with less patient dose.

\section{Methods and Materials}

Elekta Synergy (Elekta Oncology Systems Ltd, Crawley, UK) linear accelerator is designed for cone beam computed tomography based IGRT. It has a kilo voltage X-ray source (Eureka Rad-92, Varian Sapphire Housing) and amorphous silicon flat panel imagers, both mounted orthogonal to the treatment head. This $\mathrm{kV}$ imaging system is named as XVI by Elekta. XVI system allows the width and length of the $\mathrm{kV}$ $\mathrm{X}$-ray field to be selected. The width refers to the field of view (FOV). Planar images can be acquired with three different field of views namely small-S, medium-M and large-L. The difference between them is the offset from the $\mathrm{kV}$ central axis. The cone diameter incident on the detector is same for all FOV but the X-ray beam differs in inclination with difference in $\mathrm{kV}$ collimator choices. Each collimator cassette contains a lead insert with an aperture of a particular size at a particular position.

The width of the field in the couch up-down direction is called the nominal irradiated length at iso centre. There are choices in which 20 with nominal $276.7 \mathrm{~mm}$ and 10 with nominal $135.4 \mathrm{~mm}$. Collimators are labelled as M10, S20 etc. Using small FOV, volume reconstruction of up to $270 \mathrm{~mm}$ diameter is possible. To perform volumetric matching of less than $270 \mathrm{~mm}$ dia, it is preferable. A medium FOV can construct three dimensional images up to $410 \mathrm{~mm}$ diameter which is sufficient for even pelvis scan also. So, large FOV is almost not required for patient CBCT scanning. Preset information will be available for $\mathrm{kV}$ collimator selection. But; one can reconstruct the localised image to any collimator using XVI system.

15 scans of 8 different patients with ca sigmoid colon and 15 scans of 7 patients with ca lung were used in this study. The patients were immobilised with thermoplastic mask using all in one board (Orfit industries, USA). The planning CT study set, structure set and plan were exported using $\mathrm{XiO}$ release 4.33.02 planning system (Computerized Medical Systems, USA) to the XVI workstation. For both the sites, scans were taken using full gantry rotation. $\mathrm{kV}$ and $\mathrm{mAs}$ for both studies were 120 and 650 as specified in preset. Numbers of CBCT projections are same in all fields of views in full gantry rotation. The planning isocenter was taken as correction reference. The translational and rotational shifts were obtained based on this point. Sub volumes were defined by using alignment clip box to cover the entire target. Image registration will be performed in this box when using the automatic registration methods.

When the CBCT reconstructed image is moved a certain distance to fit the reference CT reconstructed images acquired for treatment planning, then it is called a "matching".
There are two types of matching. One way of matching is to define a sub volume in the image using a click box tool and choose to let an algorithm do the matching, based on either matching for high densities called bone matching or on a grayscale matching. Registration is only carried out on pixels that lie inside the sub volume. The matching for high densities (Bone match) only takes into account tissue that has 1.5 times the density of water. The Grey value mode of automatic registration matches voxel grey scale intensity values throughout the entire sub volume. The operation of this algorithm is slower than bone match, and may take several minutes for completion. The algorithm used is a grey level correction ratio technique. Another type is to do the matching manually in all three dimensions.

Bone and grey value auto matching facilities are available in the XVI system. Grey value matching is found superior compared with bone match. ${ }^{11}$ The grey scale matching uses all parts of the pre-defined volume, which takes more time. Preset information allows using collimator with medium field of view for all the sites except head and neck. But, it is not based on target shape or size. M20 collimator was used in both cases as specified. Shift results were evaluated. Though automatic registration method was used initially, clinician verified alignment between both reference and localised images in click box region and the final shifts were carried by minor adjustments if any, using "manual matching". Images were reconstructed to small field of view (S20 collimator). Grey value matching was used here also to find set up errors. Shift results were compared in both fields of views.

\section{Results and Discussion}

Quite useful observation was found in this study. Visualisation of reconstructed images with small field of view was good and sufficient for matching. Figures 1 and 2 are the localised cone beam CT transverse, coronal and sagittal images with M20 and S20 collimators respectively in a case of ca sigmoid colon. Similarly, Figures 3 and 4 are the CBCT images of M20 and S20 in a ca lung scan. Tables 1 and 2 give the translational and rotational shift results with M20 and S20 in the case of sigmoid colon. Similarly Table $\mathbf{3}$ and $\mathbf{4}$ give the shift results of lung case. X-lateral shift, Y-longitudinal shift and Z-vertical shift were indicated in centimeters in all tables. Variations in treatment set up errors with M20 and S20 collimators were $\leq 0.2 \mathrm{~mm}$ (Table 1 and 3 ) in all the measurements which includes all the $\mathrm{x}, \mathrm{y}$ and $\mathrm{z}$ directions. Maximum variation in rotational shifts was $0.3^{\circ}$ (Table 2 and 4). Without much analysis, one can state that, these differences are negligible.

Results were found almost same with both fields of views. This is due to the fact that, auto matching do not depends on field of view selection. The matching procedure is limited to the sub volume defined prior to the registration. We generally select the total target volume within the clip box prior to 
the reference image registration. Therefore, poor reconstruction of the other structures which are away from the target volume will not affect the registration process. So, it is clear that, shift results are not affected by field of view selection. Reconstructed volume is less in small field of view when compared with other FOV s because of less scan volume. Obviously, absorbed dose will be less with this FOV. So; we did CBCT procedure for remaining fractions by using $\mathrm{kV}$ collimator with small field of view. Same preset parameters were used as in the medium FOV.
More scan volume will be exposed to radiation in medium or large field of views when compared with small field of view. Since addition of dose to the area wider than target zone may increase the probability of secondary cancers risk ${ }^{9}$, it is better to use the methods where scan area is less. Other interesting observation is, one can put any $\mathrm{kV}$ collimator irrespective of the preset, as there is no interlock/indication. Now, it is proved that, collimator is very important in the reconstruction process with different field of views. Wrong filter insertion may demand repeat scanning.

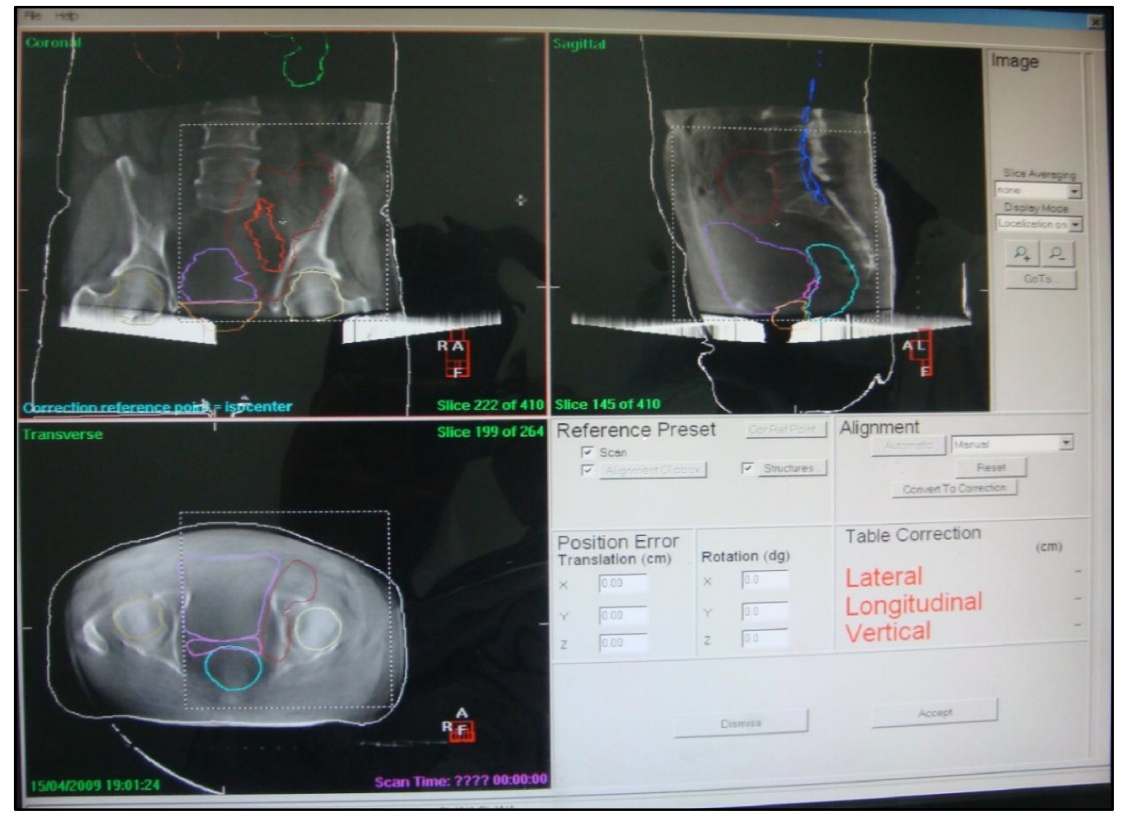

FIG. 1: Localised cone beam CT transverse, coronal and sagittal images with M20 collimator in a case of ca sigmoid colon.

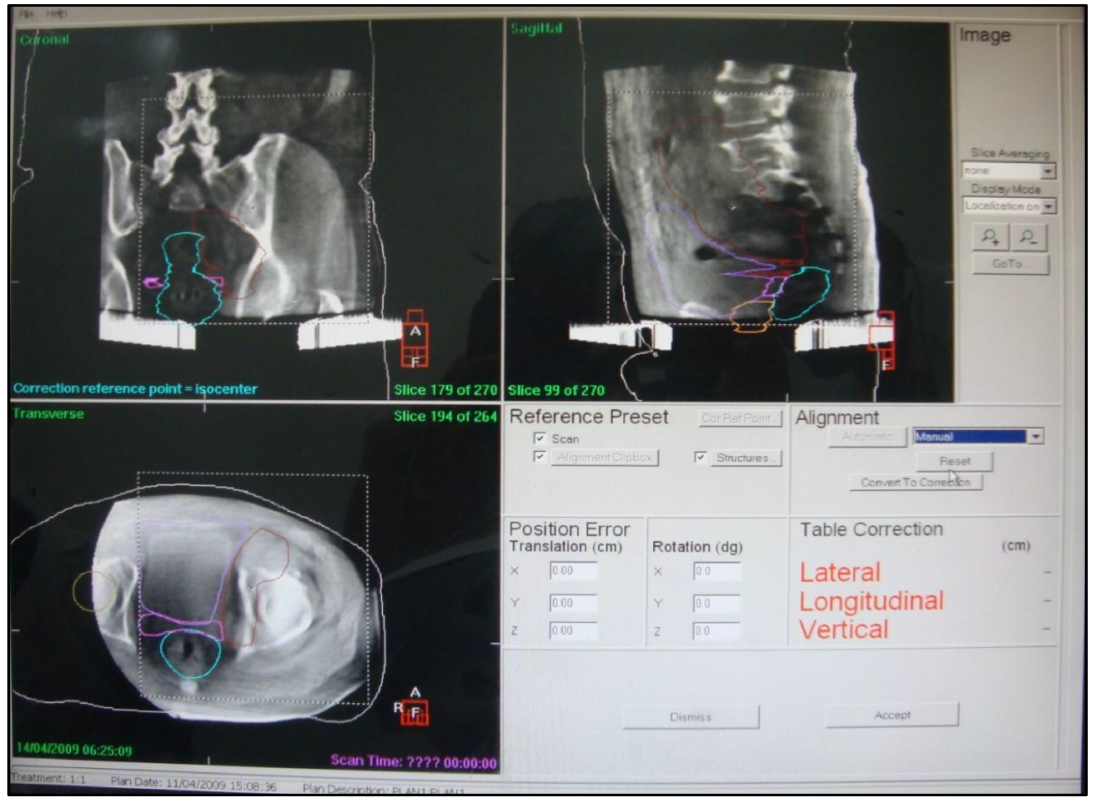

FIG. 2: Localized cone beam CT transverse, coronal and sagittal images with S20 collimator in a case of ca sigmoid colon. 
TABLE 1: Comparison of translational shift results in case of ca sigmoid colon with M20 and S20 kV collimators.(X-lateral shift,Y-longitudinal shift and Z-vertical shift were indicated in $\mathrm{cms}$ ).

\begin{tabular}{lccc:ccc}
\hline \hline Scan No & \multicolumn{3}{c}{ M20 collimator } & \multicolumn{3}{c}{ S20 collimator } \\
\hline & $\mathrm{X}$ & $\mathrm{Y}$ & $\mathrm{Z}$ & $\mathrm{X}$ & $\mathrm{Y}$ & $\mathrm{Z}$ \\
\cline { 2 - 7 } 1 & -0.14 & -0.23 & 0.32 & -0.17 & -0.23 & 0.32 \\
2 & -0.29 & 0.44 & -0.15 & -0.28 & 0.45 & -0.15 \\
3 & 0.35 & -0.21 & 0.18 & 0.35 & -0.21 & 0.20 \\
4 & 0.27 & 0.50 & -0.25 & 0.27 & 0.49 & -0.27 \\
5 & 0.39 & -0.41 & 0.30 & 0.39 & -0.41 & 0.30 \\
6 & 0.27 & 0.72 & 0.29 & 0.27 & 0.74 & 0.28 \\
7 & -0.25 & -0.33 & 0.10 & -0.27 & -0.35 & 0.09 \\
8 & -0.15 & 0.55 & -0.03 & -0.14 & 0.57 & -0.02 \\
9 & 0.14 & 0.66 & -0.26 & 0.14 & 0.66 & -0.26 \\
10 & 0.06 & 0.53 & 0.33 & 0.05 & 0.53 & 0.33 \\
11 & 0.09 & 0.61 & -0.03 & 0.09 & 0.62 & -0.03 \\
12 & 0.08 & 0.64 & -0.18 & 0.08 & 0.64 & -0.18 \\
13 & 0.10 & 0.75 & 0.32 & 0.09 & 0.75 & 0.33 \\
14 & 0.20 & 0.72 & -0.05 & 0.20 & 0.72 & -0.05 \\
15 & -0.32 & -0.11 & 0.27 & -0.30 & -0.12 & 0.29 \\
\hline \hline
\end{tabular}

TABLE 2: Comparison of rotational shift results in case of ca sigmoid colon with M20 and S20 kV collimators.

\begin{tabular}{lccc:ccc}
\hline \hline Scan No & \multicolumn{3}{c}{ M20 collimator } & \multicolumn{3}{c}{ S20 collimator } \\
\hline & $\mathrm{X}^{0}$ & $\mathrm{Y}^{0}$ & $\mathrm{Z}^{0}$ & $\mathrm{X}^{0}$ & $\mathrm{Y}^{0}$ & $\mathrm{Z}^{0}$ \\
\cline { 2 - 8 } 1 & 359 & 0.5 & 0.2 & 359 & 0.5 & 0.3 \\
2 & 1.7 & 359.0 & 0.2 & 1.7 & 359.0 & 0.2 \\
3 & 358.6 & 0 & 1.2 & 358.6 & 0.2 & 1.2 \\
4 & 2.2 & 359.9 & 0.0 & 2.1 & 0.1 & 0.1 \\
5 & 1.1 & 0.9 & 3 & 1.1 & 0.9 & 2.8 \\
6 & 1.6 & 358.3 & 358.7 & 1.5 & 358.2 & 358.6 \\
7 & 358.3 & 1.7 & 0.3 & 358.5 & 1.6 & 0.3 \\
8 & 1.7 & 358.3 & 0.1 & 1.7 & 358.3 & 0.2 \\
9 & 359.1 & 0.5 & 2.1 & 359.0 & 0.5 & 2 \\
10 & 1.2 & 358.3 & 0.1 & 1.2 & 358.2 & 0.1 \\
11 & 1.5 & 359.3 & 359.8 & 1.6 & 359.3 & 359.8 \\
12 & 359 & 1.7 & 359.5 & 359.2 & 1.7 & 359.5 \\
13 & 1.9 & 357.7 & 358.5 & 1.9 & 357.7 & 358.5 \\
14 & 357.8 & 2 & 358.7 & 357.9 & 2 & 358.7 \\
15 & 1.0 & 358.2 & 2.0 & 0.9 & 358.2 & 2.0 \\
\hline \hline
\end{tabular}

TABLE 3: Comparison of translational shift results in case of ca lung with M20 and S20 kV collimators.

\begin{tabular}{lccc|ccc}
\hline \hline Scan No & \multicolumn{3}{c}{ M20 collimator } & \multicolumn{3}{c}{ S20 collimator } \\
\hline & $\mathrm{X}$ & $\mathrm{Y}$ & $\mathrm{Z}$ & $\mathrm{X}$ & $\mathrm{Y}$ & $\mathrm{Z}$ \\
\cline { 2 - 7 } 1 & 0.51 & -0.55 & -1.18 & 0.51 & -0.56 & -1.17 \\
2 & 0.32 & 0.15 & 0.11 & 0.32 & 0.15 & 0.11 \\
3 & 0.29 & 0.61 & -0.93 & 0.30 & 0.63 & -0.93 \\
4 & -0.28 & 0.45 & 0.66 & -0.28 & 0.45 & 0.65 \\
5 & 0.41 & -0.39 & 0.91 & 0.40 & -0.39 & 0.91 \\
6 & -0.27 & -0.71 & 0.38 & -0.25 & -0.71 & 0.38 \\
7 & 0.64 & 0.43 & -0.21 & 0.64 & 0.43 & -0.21 \\
8 & 0.58 & -0.28 & 0.95 & 0.58 & -0.28 & 0.95 \\
9 & -0.43 & -0.52 & -1.19 & -0.41 & -0.52 & -1.19 \\
10 & 0.56 & 0.33 & -1.18 & 0.56 & 0.32 & -1.18 \\
11 & 0.45 & -0.32 & 0.61 & 0.45 & -0.33 & 0.61 \\
12 & 0.55 & 0.51 & -0.41 & 0.54 & 0.51 & -0.41 \\
13 & -0.33 & -0.45 & 0.59 & -0.34 & 0.45 & 0.59 \\
14 & -0.38 & 0.49 & -0.37 & -0.37 & 0.50 & -0.37 \\
15 & 0.21 & -0.33 & -0.50 & 0.21 & -0.35 & -0.51 \\
\hline \hline
\end{tabular}


Table 4: Comparison of rotational shift results in case of ca lung with M20 and S20 kV collimators.

\begin{tabular}{lccc|ccc}
\hline \hline Scan No & \multicolumn{3}{c}{ M20 collimator } & \multicolumn{3}{c}{ S20 collimator } \\
\hline & $\mathrm{X}^{0}$ & $\mathrm{Y}^{0}$ & $\mathrm{Z}^{0}$ & $\mathrm{X}^{0}$ & $\mathrm{Y}^{0}$ & $\mathrm{Z}^{0}$ \\
\cline { 2 - 7 } 1 & 4.3 & 359.8 & 358.3 & 4.3 & 359.8 & 358.1 \\
2 & 358 & 2.3 & 2.4 & 358 & 2.3 & 2.4 \\
3 & 3.2 & 358.8 & 0.2 & 3.2 & 358.8 & 0.2 \\
4 & 0.6 & 359.5 & 1.4 & 0.6 & 359.5 & 1.4 \\
5 & 2.9 & 356.0 & 358.3 & 3.0 & 356.0 & 358.3 \\
6 & 358.8 & 0.9 & 2.1 & 358.8 & 1 & 1.9 \\
7 & 4.7 & 357.3 & 357.7 & 4.5 & 357.3 & 357.7 \\
8 & 1.2 & 1.7 & 0.8 & 1.2 & 1.7 & 0.8 \\
9 & 4.3 & 359.9 & 356.9 & 4.3 & 359.8 & 357.2 \\
10 & 4.0 & 358.0 & 358.1 & 4.3 & 358.0 & 358.1 \\
11 & 359.1 & 3.7 & 358.0 & 359.1 & 3.8 & 358.0 \\
12 & 4.5 & 358.7 & 357.5 & 4.6 & 358.9 & 357.5 \\
13 & 358.9 & 3.3 & 359.0 & 359 & 3.3 & 359.1 \\
14 & 3.1 & 359.5 & 0.7 & 3.1 & 359.8 & 0.5 \\
15 & 359.7 & 359 & 0.9 & 359.5 & 359.1 & 0.9 \\
\hline \hline
\end{tabular}

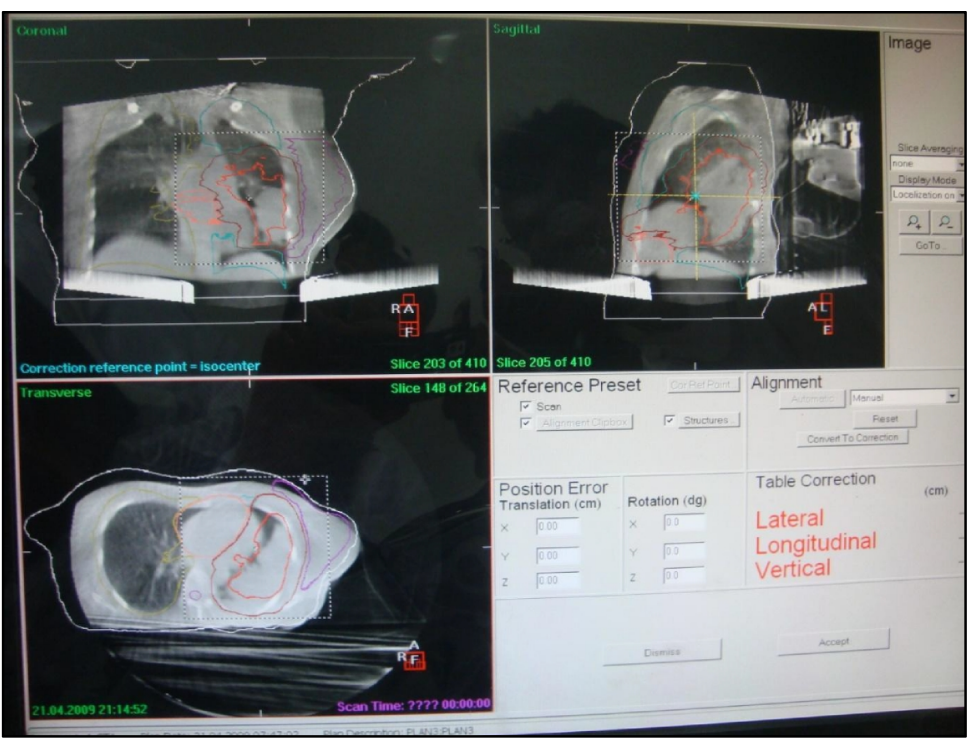

FIG. 3: CBCT transverse, coronal and sagittal images of M20 collimator in a ca lung scan.

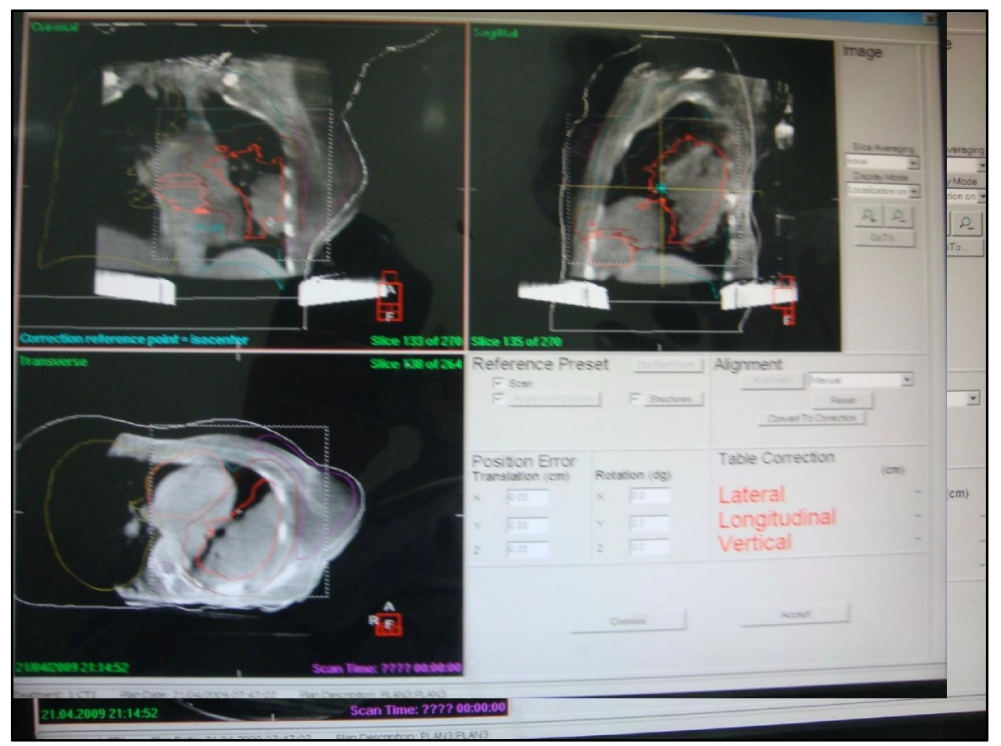

FIG. 4: CBCT transverse, coronal and sagittal images of S20 collimator in a ca lung scan. 


\section{Conclusion}

It was found that, imaging with S20 (Small field of view) collimator gave same shift results comparable to M20 (Medium field of view) in both the cases of sigmoid colon and lung tumour volumes of all selected patients. But, tumour shapes and sizes will be changed from patient to patient. Small field of view may not be sufficient always. So, it is suggested that, small field of view can be used wherever possible after verification in the first fraction of treatment to reduce scan volume. As $\mathrm{kV}$ collimator plays important role in the reconstruction process, there should be interlock to prevent wrong collimator selection.

\section{Conflict of interest}

The authors declare that they have no conflicts of interest. The authors alone are responsible for the content and writing of the paper.

\section{References}

1. Jassal K, Munshi A, Sarkar B, et al. Validation of an integrated patient positioning system: Exactrac and iViewGT on Synergy Platform. Int J Cancer Ther Oncol 2014; 2:020212.

2. Alaei P, Spezi E. Commissioning kilovoltage cone-beam CT beams in a radiation therapy treatment planning system. J Appl Clin Med Phys 2012; 13:3971.

3. Huang Q, Zeng GL, You J, Gullberg GT. An FDK-like cone-beam SPECT reconstruction algorithm for non-uniform attenuated projections acquired using a circular trajectory. Phys Med Biol 2005; 50:2329-39.

4. Cho PS, Johnson RH, Griffin TW. Cone-beam CT for radiotherapy applications. Phys Med Biol 1995; 40:1863-83.

5. Ghilezan M, Yan D, Liang J, et al. Online image-guided intensity-modulated radiotherapy for prostate cancer: How much improvement can we expect? A theoretical assessment of clinical benefits and potential dose escalation by improving precision and accuracy of radiation delivery. Int J Radiat Oncol Biol Phys 2004; 60:1602-10.

6. Oldham M, Létourneau D, Watt L, et al. Cone-beam-CT guided radiation therapy: A model for on-line application. Radio ther Oncol 2005; 75:271-8.

7. Jaffray DA. Emergent technologies for 3-dimensional image-guided radiationdelivery. Semin Radiat Oncol 2005; 15:208-16.
8. Yan D, Lockman D, Martinez A, et al. Computed tomography guided management of interfractional patient variation. Semin Radiat Oncol 2005; 15:168-79.

9. Amer A, Marchant T, Sykes J, et al. Imaging doses from the Elekta Synergy X-ray cone beam CT system. Br J Radiol 2007; 80:476-82.

10. Kan MW, Leung LH, Wong W, Lam N. Radiation dose from cone beam computed tomography for image-guided radiation therapy. Int J Radiat Oncol BiolPhys 2008; 70:272-9.

11. Meyer J, Wilbert J, Baier K, et al. Positioning accuracy of cone-beam computed tomography in combination with a HexaPOD robot treatment table. Int J Radiat Oncol Biol Phys 2007; 67:1220-8. 